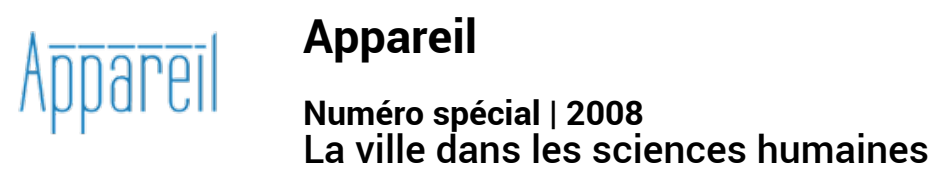

Introduction

\title{
La ville vestige
}

Jacques Boulet

\section{CpenEdition}

Journals

Édition électronique

URL : http://journals.openedition.org/appareil/445

DOI : 10.4000/appareil.445

ISSN : 2101-0714

Éditeur

MSH Paris Nord

Référence électronique

Jacques Boulet, "La ville vestige », Appareil [En ligne], Numéro spécial | 2008, mis en ligne le 30 juin 2008, consulté le 30 juillet 2020. URL : http://journals.openedition.org/appareil/445 ; DOI : https:// doi.org/10.4000/appareil.445

Ce document a été généré automatiquement le 30 juillet 2020

\section{(ब) $(\mathbb{Q} \Theta$}

Appareil est mis à disposition selon les termes de la Licence Creative Commons Attribution - Pas d'Utilisation Commerciale - Pas de Modification 4.0 International. 


\title{
Introduction
}

\section{La ville vestige}

\author{
Jacques Boulet
}

\begin{abstract}
Je parle de la ville, nouveauté d'aujourd'hui et ruine d'après-demain, enterrée chaque jour et chaque jour ressuscitée,... Je parle de la ville, bergère des siècles, mère qui nous engendre et nous dévore, nous invente et nous oublie.

Octavio Paz, Mexico, 5 mai 1986
\end{abstract}

\section{La ville inquiète $^{1}$}

1 La question urbaine reste ouverte, inquiète, en projet. L'idée même d'une maîtrise de l'urbain ne semble guère avoir de pertinence au-delà de quelques régulations partielles, préventives ou réparatrices, souvent altérées par des effets imprévus. La compréhension d'un fait social total et mondial'2, l'habiter contemporain, demeure fragmentaire: multiplication des descriptions partielles, inflations des doctrines salvatrices, désuétude accélérée des politiques de la ville, des projets et des réalisations. L'autre ville, celle d'hier, celle d'un ailleurs, celle d'un futur déjà là, voire l'autre de la ville et même la «non ville ", prennent tour à tour la figure de la promesse, de la repentance ou du désastre.

2 Fin des villes et de la ville ${ }^{3}$ ? Mort de la ville, règne de l'urbain ou de l'après ville ${ }^{4}$ ? La question de la ville, de son sens, ne se poserait donc plus qu'à partir de sa disparition ou de ses restes, ou tout simplement n'aurait plus de sens. La ville serait vestige, présence d'une absence.

3 Mais la ville est-elle vraiment mise en question ? Quelle ville tant regrettée a disparu après tant d'autres? De quelle ville parlons-nous? Quelle(s) ville(s) est en projet ou simplement émergente? Quelles villes voulons-nous? Quelles villes pouvons-nous ? Et qui est ce « nous »? De telles questions appellent des réponses projetant de nouveaux habitats s'ajoutant à la diversité, trop vite oubliée, de ce qui fut appelé "ville" au cours de l'histoire. Pourtant la ville semble moins une question de cet ordre qu'un problème, 
plutôt technique, urbanistique, de l'ordre du que faire ou du comment faire ? Que faire des villes dites « historiques »? Ou comment faire de la ville, de l'urbain et même de la ville sur la ville? La ville problème, relève désormais d'une compétence collective d'experts en habitat. Des solutions, sans cesse reprises, mettent en œuvre une rationalité vraisemblable de moyens en vue d'un mieux-être de l'habitat dit "urbain", dont la ville a été, est ou pourrait être, l'un de ses modes. Les projets de ville abondent. Mais de quelle ville s'agit-il ?

Ce que nous désignons aujourd'hui par "ville" apparaît aussi vague et divers que l'emploi des termes Ville, Stadt, Town dans la société féodale de toute l'Europe, termes qui comme le note l'historien Marc Bloch, ne permettaient pas de distinguer une ville d'un village ${ }^{5}$. De la confusion entre "ville " et "cité " nous pourrions encore nous alarmer autant que Jean-Jacques Rousseau déclarant :

La plupart prennent une ville pour une cité et un bourgeois pour un citoyen. Ils ne savent pas que les maisons font la ville, mais que les citoyens font la cité6.

Sauf que, si la ville ne fait pas le citoyen, le citoyen apparaît encore comme la condition de la ville. Alors nous parlons encore de la ville. Mais nous n'avons jamais affaire qu'à des villes, qu'à cette ville-ci ou cette ville-là, une ville toujours singulière. La ville, serait-elle un vestige, est un pluriel.

6 Signes d'inquiétude : la disparition des villes est conjurée par l'invocation à la ville. Ainsi s'explique, sans doute, la persistance du mot sans la chose, y compris dans des contre-emplois rarement ironiques, comme Grossstadt, Ville radieuse, ville nouvelle, ville monde, mégaville, Instant City, Edge city, City of bits, E-city, Ville globale, Ville générique, Ville diffuse, Exurbia, etc. Le radical polis dit encore la ville qui n'est plus : métropole, mégalopole, mégapole, métapole, etc. Les préfixes évoquent la ville-mère, la très grande ville, la ville au-delà de la ville, etc. ${ }^{7}$. La profusion des composés en « urbain », « cité » ou en " city » assortis des post-, péri-, sub-, infra-, méga-, décline la ville sur le mode de sa dégradation ou disparition. Ce qu'il advient de l'habitat ne saurait donc être nommé qu'en référence à la ville, par fragment, dérivation ou différenciation.

7 L'autre de la ville est aussi décrit à partir d'un manque ou d'un simulacre de ville: absence d'espace public, de rue, de place, d'îlot, de jardin, de cafés, de commerces, de services, de transports, bref de tout ce qui serait l'expérience ordinaire d'un vivre ensemble urbain, plutôt occidental et riche. Ajoutons aussi quelque épisode à «la guerre $d u f^{f a u x}{ }^{8} »$ : l'aventure urbaine se déroule dans un coquet morceau de ville privée, sécurisé et si possible à thème. Ou encore le désastre urbain du monde pauvre, celui de toutes les misères et violences, est posé comme paradigme du destin inéluctable, sinon de nos villes, du moins de toute urbanisation ${ }^{9}$, le pire des mondes possibles ${ }^{10}$, etc.

8 Parole incantatoire, la ville est alors dotée de pouvoirs magiques. C'est la ville solution. Par exemple, le pouvoir de régénérer la dissémination urbaine ou le grand ensemble, de s'opposer à la relégation sociale et à l'entre soi, d'appeler à un mélange social et à l'équité d'un partage de l'espace. Ou bien, la ville dense, remède aux gaspillages de l'étalement urbain, serait garante d'une économie durable, entendons oikomomia comme l'art de bien administrer l'habitat. 


\section{La ville problème}

9 En politique urbaine, les solutions précèdent souvent les problèmes. Par exemple, comment transformer les villes à l'aune d'une modernité toujours réaffirmée comme une nécessité ? Le problème est pourtant résolu de fait et sans aucun doute avec nombre d'effets pervers qui deviendront autant de problèmes. L'urbanisation dite " galopante » l'a fait et le fait au-delà des espérances visionnaires de toutes les avantgardes du désurbanisme ${ }^{11}$. Dissoute, la contradiction entre ville et campagne. Généralisé, le territoire des mobilités maillé des réseaux physiques et immatériels hiérarchisés ${ }^{12}$. Démultipliée, la croissance linéaire et concentrique. Outrepassée, la ville invertie et dissociée des édifices radieux posés sur le territoire. Désormais c'est plutôt le musée imaginaire des objets urbains prospectifs qui glace d'effroi ou consterne d'insignifiance. Rare est l'humour qui les aurait sauvés de l'esprit de géométrie. Anachronisme, simplification outrancière, usurpation de l'utopie, témoignent surtout de l'incapacité à décrire et re-figurer le réel urbain dans sa complexité et sa temporalité, comme l'ont fait et le font, par exemple, la littérature et le cinéma. Et cela même dans les provocations suggestives du «post-city age ${ }^{13}$ » et du «non-plan for a non city ${ }^{14}$ » où l'urbanisation tendancielle fut posée, il y a plus de quarante ans, comme le modèle de l'urbain à venir. La posture "avant-garde urbaine ", réduite au dérisoire par l'urbanisation elle-même, a presque disparu. L'idée de "ville nouvelle », du moins en occident, ne resurgit que rarement avec un profil bas. Ailleurs, il est permis de se demander de quel espoir est porteur, l'habitat nommé "ville nouvelle" qui semble surgir de nulle part comme un mirage. L'heure est à la réparation de l'urbanisation réticulée, polymorphe et polycentrée, à sa régulation au nom de l'urbain. Cette régulation, devenue l'une des techniques même de l'urbanisation, n'est-elle pas aujourd'hui la seule définition possible de l'urbanisme et du "projet urbain" en acte ?

Ou encore, autre problème, comment préserver ce qui reste des villes d'hier? Les solutions abondent. Faut-il définir la préservation, la protection, la conservation, l'adaptation de la ville monument? Les dispositifs réglementaires et institutionnels y pourvoient. Faut-il restaurer, rénover, réparer, réhabiliter, etc.? Le savoir-faire est acquis. Le problème, s'il existe, est de l'ordre du comment mieux faire, du débat sur la loi non appliquée ou sur le tri, faute de moyens ou en regard des nécessités d'intérêt général. Que préserver? Quelles mémoires ? Le conflit politique des valeurs d'usage et de mémoire, si bien analysé par Aloïs Riegl, il y a plus d'un siècle, s'avère plus que jamais d'actualité et les compromis incertains et peu explicités ${ }^{15}$. La ville devient alors cette ville historique ci, sans sa périphérie, réduite aux traces et images qui valent pour la Ville. La partie, le monument habité de la ville, s'offre comme témoignage de l'identité du tout. Trois usages spectaculaires célèbrent l'âge de la ville monument : la ville-musée pour le grand tour des ruines et des traces ; la ville-réserve pour témoigner d'un habiter vivant sur le mode d'un « cela fut »; la ville-sauvegarde pour garantir les représentations symboliques des autorités religieuses, culturelles et politiques, voire des puissances économiques. La ville est présente certes, mais au titre d'un art d'en accommoder les restes glorieux. 


\section{La ville en disparition}

11 La ville serait chose du passé, réduite à ses traces. Le sentiment d'une disparition lente, mêlé de nostalgie a chassé des discours sur la ville les convictions les plus fermes. Après la ville, bénéfique ou maléfique, promesse de la liberté et lieu de toutes les perditions, l'étalement diffus de la société urbaine : l'urbain mondialisé ou l'ère de "l'après ville ${ }^{16}$ ». Après l'urbain, après les paroxysmes de l'urbanisation et de la dévastation urbaine, un au-delà du non urbain, un établissement de non lieux traversé de multiple flux : l'ère post-urbaine ${ }^{17}$. Un cyber-monde promet une imminence communautaire, celle du village global ou de la ville planétaire, qui se jouerait sur la scène d'un espace public virtuel $^{18}$. Bref, la ville est morte, vive la ville.

12 Cette ville racontée qui tel le Phénix renaît sans cesse de ses cendres, n'est pas celle de ses divers états historiques en Europe : celle du lieu circonscrit d'une communauté politique libre avec ses multiples variations juridiques et économiques. Ce n'est pas, par exemple, la ville bourgeoise de «L'Europe des Capitales » décrite par Max Weber ${ }^{19}$ ou Julio Carlo $\operatorname{Argan}^{20}$ qui, en tant que ville, s'est donnée la représentation de son pouvoir en faisant œuvre de ses lieux et dont la violence, les échecs, les vicissitudes auraient été oubliées. Ce n'est pas non plus la ville de la littérature: le Paris de Queneau ou de Simenon, la Nantes de Gracq, la Lisbonne de Pessoa, le Dublin de Joyce, la Barcelone de Mendoza, la Buenos Aires de Borges et Sabato, Le Caire de Naguib Mahfouz, le Beyrouth d'Elias Khoury, l'Istambul de Orhan Pamuk, la New York de Jerom Charyn, etc.

Cette ville en disparition, c'est sinon la "Cité idéale», du moins celle de l'urbanité souhaitable, l'adéquation heureuse d'une société à ses lieux, la ville antérieure pleinement habitée dont les fragments visibles témoignent de l'unité disparue et originelle ${ }^{21}$. C'est la ville d'une narration qui en décrit l'avènement, les péripéties et la déchéance, avant d'annoncer sa métamorphose dans un nouvel équilibre. Une histoire au double sens de récit et de devenir. Des histoires mêlées dont la ville est à la fois l'origine et la promesse. Intrigue faible ${ }^{22}$.

14 Avec la ville en disparition, la question du sens de la ville ou d'une ville, ou de son identité qui en est une variante, doit donc être déplacée. Elle devient : quel est le sens de cette quête de sens ? Ces récits persistants de la ville en disparition répondent à leur manière, souvent assez frustre et naïve, à la question quel sens peut-il y avoir à poser la question du sens de la ville, de ses identités multiples. De quoi s'agit-il ? De l'attente du politique ou mieux du commun, d'un commun (la commune, il commune en italien). C'est la ville comme visée éthique, c'est-à-dire une allégorie des lieux de la vie bonne avec les autres, pour les autres, parmi les autres dans des institutions justes. C'est la multitude acceptée, espacée par un partage, par une division reconnue. En témoignent une inquiétude, une nostalgie et une quête d'identité.

\section{Inquiétude}

15 La ville racontée, faut-il encore y insister, n'est certes pas la polis, mais la polis y est en attente. La "mise en commun des paroles et des actes" qui selon Aristote caractérisait la polis, travaille le récit de la ville en négatif. Une forme historique de la socialité, la ville réalisée, cette figure infiniment variée d'un vouloir vivre ensemble, toujours inachevée, ne signifie plus les appartenances libres. Tout ce que la ville reliait ou visait à relier en un lieu, instaurait par son édification, semble désormais délié, dispersé. La métaphore 
d'un espace public figuré par la ville a perdu sa visibilité. Tous les termes proches dont l'institution urbaine était le creuset : la civilité, la citadinité, l'urbanité, la citoyenneté, qui tous portaient en eux-mêmes l'accueil d'autrui, qui qualifiaient le politique, semblent n'être plus invoqués que pour prendre la mesure de l'indifférence et des ségrégations sociales, voire de la disparition même du politique. La ville n'atteste plus du commun, d'une présence à soi de la communauté. Elle est, précise-t-on, une « ville à trois vitesse $"$ : relégation, périurbanisation, gentrification ${ }^{23}$. L'espace public, où Hannah Arendt décelait pour la polis, l'invention originelle d'une scène sans lieu où pouvait se jouer l'immortalité des mortels, ne pallie plus à la fragilité des actions humaines. L'espace politique ne témoigne plus de la fugacité de l'action, de l'imprévisibilité de son sens et de ses résultats (toujours caché à l'acteur et seulement dévoilés par le narrateur), en offrant l'immortalité pour des bonnes et mauvaises actions. L'espace de cette commémoration a disparu de la ville. À la célèbre phrase de l'Athénien qui délie la ville de son lieu « où que vous alliez, vous serez une polis », la ville racontée semble répondre « où que vous irez, vous trouverez la ville ».

\section{Nostalgie}

16 «Le non habiter est le caractère essentiel de la vie métropolitaine ». Massimo Cacciari ${ }^{24}$ prenait-il seulement acte d'une situation métropolitaine ou quelque trace d'un regret ne traversait-elle pas ainsi son discours? Quoi qu'il en soit, il est difficile de ne pas verser la plus grande part du discours de couleur heideggerienne, sur «l'habiter comme trait fondamental de l'être » et sur la préséance de l'habiter sur le bâtir, du côté $\mathrm{du}$ ressassement. Sans entrer ici dans une discussion de ce thème, il devrait suffire de rappeler que la célèbre conférence exotérique et polémique prononcée à Darmstadt se termine par cette remarque que le déracinement (Heimatlosigkeit) « est le seul appel qui invite les mortels à habiter ». Aussi semble-t-il plus profondément heideggerrien de continuer l'interrogation en ne dissociant pas, la demeure de l'exil et à l'instar Michel Harr de reprendre la réflexion en confrontant le célèbre poème d'Hölderlin L'homme habite en poète à ce vers du poète Saint-John Perse : «Notre site est l'inhabitable ${ }^{25}$ ». L'appel à habiter n'en sous-tend pas moins le récit de la ville en disparition. Le spectre métropolitain d'une prolifération urbaine indifférenciée, sans limites, justifie tous les retours à l'origine, avant le mal ou la chute. Le retour à l'espace d'origine, à la ville antérieure au chaos, à ses formes types et à ses règles génératives, effacerait le temps du déclin. La réversibilité dans l'espace, la possible réitération des lieux, se jouerait ainsi de l'irréversibilité du temps. Le dispositif de la nostalgie, tel que le décrit Vladimir Jankélévitch, est alors en place ${ }^{26}$. La déception inéluctable.

\section{Quête d'identité}

17 La perte et la quête d'identité nourrissent aussi abondamment les récits de la ville en renvoyant à la question du sens, à la délimitation du propre de telle ville. L'identité d'une ville serait son nom propre, sa différence, sa singularité, ou du moins celle qui fait sens. Thème équivoque, puisqu'il s'agit à la fois de dire et projeter le même de telle ville, l'identique dans le temps, et l'autre ville qu'elle devient. Thème omniprésent, puisqu'il détermine la plus grande part des prescriptions architecturales et urbaines inscrites dans les règlements d'urbanisme. 
Personne ne doute cependant de la singularité des villes, de leur unicité. Chacun peut en reconnaître les signes discrets. La prétendue uniformité envahissante de leur périphérie témoigne surtout d'un défaut d'expérience ou d'observation. Pourtant, les villes, signe d'une perte de sens, sont en quelque sorte sommées de décliner leur identité. Une sorte de police urbanistique, entre autres, y veille avec les meilleures intentions réglementaires ou projectuelles: photo, nom, prénoms, taille, signes particuliers. La préservation ou l'affirmation de l'identité répondrait à une disparition de l'individualité. Le pire, semble-t-il, serait qu'une ville puisse se faire passer pour une autre ou avoir une existence clandestine. Ou qu'elle possède plusieurs identités : des faux papiers peut-être, ou plusieurs états civils, par exemple, Paris alias Las Vegas !

Pourtant l'expérience de la littérature et du cinéma est sur ce point irremplaçable pour attester que le sens d'une ville n'est jamais clos et ne peut se résorber dans l'identité d'un soi propre.

20 Le sens d'une ville court, entre autres, à travers l'infinité des récits de ville, des intrigues brisées par l'inédit, l'inouï de l'histoire. Il ne surgit qu'en tant qu'ouverture, là où rien d'essentiel n'est acquis, ni déposé mais advient seulement. Le sens d'une ville se livre dans la fragilité de sa venue. Cette ouverture suspend la possibilité du même immuable, de l'identique, (la mêmeté de l'identité-idem selon Paul Ricœur), et libère le même en devenir du soi-même, (l'ipséité de l'identité-ipse). La ville est cette ville, singulière, différente, non parce qu'elle resterait identique à elle-même en opposition à quelque autre (alterum), la périphérie, la désurbanisation, les autres villes, etc. Toute ville n'est elle-même que par ses différenciations internes, que parce qu'elle est vraiment autre (aliud), c'est-à-dire quelque chose d'autre en un sens indéterminé. Ce jeu ouvert du même et de l'autre de la ville, appartient à l'expérience commune de flânerie, qui laisse venir le sens, l'étonnement dans la distraction : la ville qui apparaît toujours autre parce qu'elle est la même, et toujours la même parce qu'elle est autre. Ce qui laisse deviner les raisons de l'échec de toutes les répétitions du même, de l'identité close, qui président au sauvetage de la ville monument, et donc travaille à son oubli.

De quels périls, note Walter Benjamin, les phénomènes sont-ils sauvés? Pas seulement du discrédit et mépris dans lequel ils sont tombés, mais de la catastrophe que représente une certaine façon de les transmettre en les célébrant comme " patrimoine ». Ils sont sauvés lorsqu'on met en évidence chez eux la fêlure. Il y a une tradition qui est catastrophe ${ }^{27}$.

Que reste-t-il de la ville et que réactive sans cesse les récits (y compris nos projets les meilleurs) ? Très précisément, un vestige (de vestigium, empreinte du pas), non pas la trace d'une absence, le reste témoignant de la ville, de son idée, mais la présence de son passage, la trace de sa venue, le rythme intermittent de sa présence et de son absence, de son apparition et sa disparition. Un vestige, concept vague, comme le souligne JeanLuc Nancy, de quelque chose entre présence et absence, entre tout et rien et surtout entre image et idée ${ }^{28}$. La ville vestige nous met sur la trace de la ville elle-même, ou simplement de quelque chose qui lui serait essentiel. Car ce qui reste est aussi ce qui résiste le plus. Plus encore, la ville ne serait-elle pas elle-même, essentiellement de l'ordre du vestige. N'aurait-elle jamais été que vestige? 


\section{BIBLIOGRAPHIE}

Argan Giulio Carlo, L'Europe des capitales 1600-1700, Genève, Skira, 1964.

Bailly Jean-Christophe, « La ville hors les murs », in La Ville inquiète, Gallimard (Le temps de la réflexion), 1987.

Banham Reyner, Barker Paul, Price Cédric, Hall Peter, « Non-plan: an experiment in freedom », New Society, $\mathrm{n}^{\circ 0} 26,1969$, p. 435-443.

Banham Reyner, Los Angeles, The Architecture of Four Ecologies, London, Allen Lane, 1971.

Benjamin Walter, Paris, capitale du XIX $X^{e}$ siècle, Paris, Éditions du Cerf, 1989.

Biget Jean-Louis, Hervé Jean-Claude, Thébert Yvon (dir.), Panoramas urbains : Situation de l'histoire des villes, Fontenay-aux-Roses/Saint-Cloud-Paris, ENS Éditions, 1995.

Bloch Marc, La société féodale. Les classes et le gouvernement des hommes, Paris, Albin Michel, 1940.

Boulet Jacques, « Cyberfictions, remarques », in Marcello Pazzaglini (dir.), Architetture et paesaggi della città telematica, Mancosure Editore, 2004, p. 54-67.

Cacciari Massimo, «Eupalinos ou l'architecte », Communications, 1987.

Castells Manuel, La question urbaine, Paris, François Maspéro, 1972.

Castells Manuel, La société en réseaux, Paris, Fayard, 2001.

Cauquelin Anne, «La ville-fragment », in La Ville inquiète, Gallimard (Le temps de la réflexion), 1987.

Choay Françoise, «Le règne de l'urbain et la mort de la ville », in La ville, art et architecture en Europe, 1870-1993, Paris, Centre Georges Pompidou, 1994.

Choay Françoise, « Post-urbain », in Dictionnaire de l'urbanisme et de l'aménagement, Paris, PUF, 1996.

Corboz André, « Non-city revisited », in La Ville inquiète, Gallimard (Le temps de la réflexion), 1987.

Davis Mike, Mailhos Jacques, Le pire des mondes possibles de l'explosion urbaine au bidonville global, Paris, La Découverte, 2006.

Deguy Michel, « La vraie ville est absente », in La Ville inquiète, Gallimard (Le temps de la réflexion), 1987.

Donzelot Jacques, « La ville à trois vitesse : relégation, périurbanisation, gentrification », Esprit, mars-avril 2004.

Dutour Thierry, « La mondialisation, une aventure urbaine. Du Moyen Âge au Global-blabla », Vingtième siècle. Revue d'histoire : Révolution urbaine et mondialisation, ${ }^{\circ \circ} 81$, janvier-mars 2004 , Paris, Presses de Sciences-Po.

Eco Umberto, La guerre du faux, Paris, Livre de poche, 1987.

Haar Michel, « La demeure et l'exil », in « Les symboles du lieu », Cahier de l'Herne, nº 44, 1983.

Jankélévitch Vladimir, L’irréversible et la nostalgie, Paris, Flammarion, 1983.

Joseph Isaac, La Ville sans qualités, La Tour d'Aigues, Éditions de l'Aube, 1998. 
Koolhaas Rem, New York Délire, Paris, Éditions du Chêne, 1978.

Koolhaas Rem, « La ville générique » [1994], in Mutations, Bordeaux, Actar, Arc en rêve/Centre d'architecture, 2000.

Kopp Anatole, Ville et révolution, Paris, Éditions Anthropos, 1967.

Lefèvre Henri, « La ville et l'urbain », Espaces et Sociétés, 1972.

Lepetit Bernard, « La ville : cadre, objet, sujet. Vingt ans de recherche française en histoire urbaine ", Enquête : Anthropologie, histoire, sociologie, n 4, Marseille, Parenthèses, 1996.

Lepetit Bernard, Topalov Christian (dir.), La ville des sciences sociales, Paris, Belin, 2001.

Lévi-Strauss Claude, Tristes tropiques, Paris, Plon, 1955.

Levy Jacques, «Territoire et réseaux », in Thierry Paquot (dir.), Le Monde des villes. Panorama urbain de la planète, Bruxelles, Complexe, 1996.

Mitchell William J., City of Bits: Space,Place, and the Infobahn, Londres, MIT Press, 1995.

Nancy Jean-Luc, « Au loin, Los Angeles », in La Ville inquiète, Gallimard (Le temps de la réflexion), 1987.

Nancy Jean-Luc, « Le vestige de l'art », in Les Muses, Paris, Galilée, 1994, p. 133-159.

Paquot Thierry (dir.), Le monde des villes. Panorama urbain de la planète, Bruxelles, Éditions

Complexes, 1996.

Paz Octavio, «Je parle de la ville », in La Ville inquiète, Gallimard (Le temps de la réflexion), 1987.

Préel Bernard, «L'Après-ville », in Thierry Paquot (dir.), Le monde des villes. Panorama urbain de la planète, Bruxelles, Éditions Complexes, 1996.

Ricoeur Paul, « Architecture et narrativité », Urbanisme, novembre-décécembre 1998, p. 44-51.

Riegl Alois, Le culte moderne des monuments, [1984], Jacques Boulet (intro. et trad.), Paris, l'Harmattan, 2003.

Rousseau Jean-Jacques, Du contrat social, Paris, Flammarion, 2001.

Secchi Bernado, Il raconto urbanistico, Turin, Einaudi, 1984.

Spengler Oswald, Le déclin de l'Occident, Paris, Gallimard, 1976.

Taut Bruno, Die Auflösung der Städte, Hagen,Volkwaang Verlag, 1920.

Tönnies Ferdinand, Communauté et société [1887], Paris, PUF, 1944.

«Villes-mondes, villes monstres? ?, Raisons politiques, $\mathrm{n}^{\circ 0} 15$, Paris, Presses de Sciences-Po, août 2004

Webber Melvin M., « The post-city age », Daedalus, New York, automne 1968.

Weber Max, La ville, Paris, Aubier Montaigne, 1984.

\section{NOTES}

1. La Ville inquiète, Gallimard (Le temps de la réflexion), 1987, VIII. Nous renvoyons ici à cet excellent numéro d'une revue disparue, principalement pour les articles de Jean-Christophe Bailly, « La ville hors les murs », Anne Cauquelin, « La ville-fragment », André Corboz, « Non-city 
revisited », Michel Deguy, «La vraie ville est absente », Jean-Luc Nancy, « Au loin, Los Angeles », Octavio Paz, « Je parle de la ville».

2. Pour un aperçu de l'état des études urbaines où il faudrait distinguer l'étude de la partie qui se donne pour le tout de la ville ou de l'urbain, de celle du fragment qui est construit pour valoir du tout et de celle qui espère qu'un jour l'addition des parties donnera le sens du tout ou ne s'en préoccupe peu, voir Bernard Lepetit, Christian Topalov (dir.), La ville des sciences sociales, Paris, Belin, 2001; Thierry Paquot (dir.), Le monde des villes. Panorama urbain de la planète, Bruxelles, Éditions Complexes, 1996 ; Bernard Lepetit, « La ville : cadre, objet, sujet. Vingt ans de recherche française en histoire urbaine ", Enquête: Anthropologie, histoire, sociologie, $\mathrm{n}^{\circ} 4$, Marseille, Parenthèses, 1996 ; Jean-Louis Biget, Jean-Claude Hervé, Yvon Thébert (dir.), Panoramas urbains : Situation de l'histoire des villes, Fontenay-aux-Roses/Saint-Cloud-Paris, ENS Éditions, 1995.

3. La littérature des fins annoncées est inépuisable. La fin de la ville en est souvent l'une des figures. On en rappellera ici, l'une des origines qui a profondément marqué l'émergence d'une sociologie de l'urbain, Ferdinand Tönnies, Communauté et société [1887], Paris, PUF, 1944, p. 231-240 ; Oswald Spengler, Le déclin de l'Occident, (t. I, 1918 ; t. II, 1922), Paris, Gallimard, 1976, p. 84-99. On en rapprochera utilement l'ouvrage de l'architecte, Bruno Taut, Die Auflösung der Städte, Hagen, Volkwaang Verlag, 1920.

4. Le thème de la dissociation ville/urbain est caractéristique de la fin des années 60. Pour un surpassement dialectique de la ville par l'urbain, sorte d'aufheben hégelien qui donne à penser en même temps la suppression et la conservation de la ville, à l'horizon d'une " révolution urbaine » annonçant la fin de l'Histoire, voir Henri Lefèvre, «La ville et l'urbain », Espaces et Sociétés, 1972, résumé des thèses développées dans le Droit à la ville (1968), Du rural à l'urbain (1970), La révolution urbaine (1970). Pour une critique des thèses de H. Lefèvre et un rappel à une orthodoxie marxiste procédant de la même dissociation ville/urbain et du dépassement de la «notion idéologique d'urbanisation » par une théorie de «la production sociale des formes spatiales ", voir Manuel Castells, La question urbaine, Paris, François Maspéro, 1972, p. 117-128; pour une reprise du thème de "l'urbanisation généralisée » au titre d'une évidence empirique et d'un phénomène indifférencié en Europe, voir Françoise Choay, «Le règne de l'urbain et la mort de la ville », in La ville, art et architecture en Europe, 1870-1993, Paris, Centre Georges Pompidou, 1994, p. 26-35. Les thèmes de «l'après ville » et du " post-urbain » ne sont pas toujours confondus : voir Melvin $\mathrm{M}$. Webber, "The post-city age », Daedalus, New York, automn, 1968 ; Bernard Préel, "L'Aprèsville ", in Thierry Paquot (dir.), Le monde des villes. Panorama urbain de la planète, p. 673-687. Pour une mise en perspective historique et critique de ce thème : Thierry Dutour, « La mondialisation, une aventure urbaine. Du Moyen Âge au Global-blabla», Vingtième siècle. Revue d'histoire: Révolution urbaine et mondialisation, $\mathrm{n}^{\circ 0} 81$, janvier-mars 2004, Paris, Presses de Sciences-Po. La pauvreté des récits de la ville comparés à ceux de la littérature. Bernado Secchi, Il raconto urbanistico, Turin, Einaudi, 1984.

5. Marc Bloch, La société féodale. Les classes et le gouvernement des hommes, Paris, Albin Michel, 1940, p. 112.

6. Jean-Jacques Rousseau, Du contrat social, Livre I, chap. 6, note p. 57, Paris, Flammarion, 2001.

7. Jacques Levy, «Territoire et réseaux ", in Thierry Paquot (dir.), Le Monde des villes. Panorama urbain de la planète, Paris, Complexe, 1996. On notera la tentative d'introduire une métrique pour mettre un peu d'ordre dans la profusion et l'usage des termes. Dans un phénomène général de métropolisation, l'auteur distingue trois échelles : pour exemple, les métropoles (Tokyo) les mégalopoles dont les mégapoles (Tokyo/Osaka) et les gigapoles (Koryama/Fukuoka). Sur cette base, il construit deux types idéaux de processus de métropolisation en cours : Edge city (cf. la ville lisière, Garreau, 1991), habitat diffus et polycentrique, et métropolis, ville dense centre et sa périphérie.

8. Umberto Eco, La guerre du faux, Paris, Livre de poche, 1987. 
9. Rem Koolhaas, «La ville générique » [1994], in Mutations, Bordeaux, Actar, Arc en rêve/Centre d'architecture, 2000, p. 721-742. La « ville générique », c'est la ville indifférenciée qui n'est plus la ville, l'urbanisation généralisée qui n'est plus urbaine mais plutôt tropicale, africaine ou asiatique. Paradoxes ironiques d'un contre manifeste pour des architectures célibataires (ce que ne fait pas cet architecte) et qui fait suite au «manifeste rétro-actif pour Manhattan », manifeste d'avant-garde écrit a posteriori : New York Délire, Éditions du Chêne, Paris, 1978. Pour une approche informée des "villes-monde », terme semble-t-il consacré mais aussi peu différenciant que celui de «bidonville », voir Villes-mondes, villes monstres?, Raisons politiques, ${ }^{\circ}{ }^{\circ} 15$, Paris, Presses de Sciences-Po, août 2004. On peut encore se référer au constat d'inadéquation de l'idée occidentale de ville d'un anthropologue parcourant les villes d'Inde, Claude Lévi-Strauss, Tristes tropiques, Paris, Plon, 1955, p. 151-161.

10. Mike Davis, Jacques Mailhos, Le pire des mondes possibles de l'explosion urbaine au bidonville global, Paris, La Découverte, 2006.

11. Pour une analyse éclairante de la tradition anti-urbaine nord américaine (T.A. Jefferson, Thoreau, H. Adam, H. James, entre autres) qu'il faut prolonger jusqu'aux architectes entre autres, L. Sullivan et F.L.Wright ("Broadacre City»), Morton and Lucia White, "The American Intellectual versus the American Cities », in The future Metropolis, New York, Braziller, 1961. Pour l'opposition entre « urbanistes et désurbanistes » en URSS dans les années vingt, on se réfèrera encore utilement à Anatole Kopp, Ville et révolution, Paris, Éditions Anthropos, 1967, p. 207-229.

12. Manuel Castells, La société en réseaux, Paris, Fayard, 2001.

13. Melvin M Webber, " The post-city age ».

14. Reyner Banham, Paul Barker, Cédric Price, Peter Hall, «Non-plan: an experiment in freedom», New Society, $\mathrm{n}^{\circ 0} 26,1969$, p. 435-443 ; André Corboz, "Non city revisited». André Corboz cite cette remarque de Reyner Banham, par ailleurs cycliste : « À l'instar des générations précédentes d'intellectuels qui apprirent l'italien pour lire Dante dans le texte, j'ai appris à conduire pour lire Los Angeles dans l'original». (Los Angeles, The Architecture of Four Ecologies, London, Allen Lane, 1971).

15. Alois Riegl, Le culte moderne des monuments, [1984], Jacques Boulet (intro. et trad.), Paris, l'Harmattan, 2003.

16. Bernard Préel, «L'après ville », in Thierry Paquot (dir.), Le monde des villes. Panorama urbain de la planète.

17. Melvin Webber, «The Post-cityAge »; Françoise Choay, article " Post-urbain », in Dictionnaire de l'urbanisme et de l'aménagement, Paris, PUF, 1996, donne cette définition supposée "éclairante » de l'urbain comme nouvelle civilisation: "système opératoire valable et développable en tous lieux, constitués par des réseaux matériels et immatériels ainsi que par des objets techniques et dont la manipulation retentit dans un circuit bouclé sur les rapports que notre société entretient avec l'espace, le temps et les hommes ».

18. William J. Mitchell, City of Bits: Space,Place, and the Infobahn, Londres, MIT Press, 1995.

Jacques Boulet, "Cyberfictions, remarques », in Marcello Pazzaglini (dir.), Architetture et paesaggi della città telematica, Mancosure Editore, 2004, p. 54-67.

19. Max Weber, La ville, Paris, Aubier Montaigne, 1984.

20. Giulio Carlo Argan, L'Europe des capitales 1600-1700, Genève, Skira, 1964.

21. Dans la lignée de Max Weber, une analyse de l'idéal-type de la ville : Isaac Joseph, La Ville sans qualités, La Tour d'Aigues, Éditions de l'Aube, 1998.

22. Comparés à ceux de la littérature, l'intrigue faible des récits urbanistiques de la ville, est bien décrite par Bernado Secchi, Il raconto urbanistico, Turin, Einaudi, 1984.

23. Jacques Donzelot, «La ville à trois vitesse : relégation, périurbanisation, gentrification », Esprit, mars-avril 2004.

24. Massimo Cacciari, « Eupalinos ou l'architecte », Communications, 1987. 
25. Michel Haar, «La demeure et l'exil », in « Les symboles du lieu », Cahier de l'Herne, $\mathrm{n}^{\circ} 44,1983$; pour une mise en question subtile du thème heideggérien de la préséance de l'habiter sur le bâtir, Paul Ricoeur, "Architecture et narrativité », Urbanisme, novembre-décécembre 1998, p. 44-51.

26. Vladimir Jankélévitch, L'irréversible et la nostalgie, Paris, Flammarion, 1983.

27. Walter Benjamin, Paris, capitale du XIX ${ }^{e}$ siècle, Paris, Éditions du Cerf, 1989, p. 491.

28. Jean-Luc Nancy, « Le vestige de l'art », in Les Muses, Paris, Galilée, 1994, p. 133-159.

\section{AUTEUR}

\section{JACQUES BOULET}

Architecte, Professeur à l'ENSAPLV 